\title{
Performance of Ad hoc Network in Mixing of Omni and Directional Mode Antenna
}

\author{
Asrar Ahmad Ansari \\ College of e-Learning \& \\ Distance Learning Deanship \\ King Saud University, Riyadh \\ Saudi Arabia- 11485
}

\author{
Tauseef Ahmad \\ E- Learning Unit \\ Department of Biochemistry \\ King Saud University, Riyadh \\ Saudi Arabia- 11485 \\ Mohammad Serajuddin \\ College of e-Learning \& Distance Learning Deanship \\ King Saud University, Riyadh, Saudi Arabia
}

\author{
Ali Akhtar \\ College of e-Learning \& \\ Distance Learning Deanship \\ King Saud University, Riyadh \\ Saudi Arabia- 11485
}

\begin{abstract}
This work discusses the performance degradation of ad hoc network because few nodes of the network utilizing omni directional antenna and some of them using directional antennas. We have many directional MAC protocols. But most of them has kept it in mind that each node of the network will have a directional antenna mounted on it. While in real time scenario it is quite unusual that a sudden change will occur and every laptop, palmtop, computer and other devices will be turned into a smart antenna holding device. There will be network scenario, where we will have devices of mixed nature, omni directional as well as directional antenna patterned. Using OPNET 14.5 simulation tool we have simulated a directional and omni mode mixed scenario with IEEE 802.11 MAC protocol. For directionality we have designed an antenna pattern of 90 degree with the help of the antenna editor of the simulation tool. We found that lack of a specific protocol for the above kind of scenario degrades the performance of ad hoc network.
\end{abstract}

\section{KEYWORDS}

Ad hoc, MAC protocol, Directional antenna, OPNET 14.5, Antenna pattern

\section{INTRODUCTION}

Advancement in smart antenna technology provides plenty of opportunity for the communication network to accomplish healthier performance output on all the traditional parameters like delay, throughput, energy consumption etc.

Beam forming using directional antenna is quite old technology but its large shape and huge cost did not appropriate it for small network. It was by and large used for application like, RADAR, SONAR, Imaging, Geophysical exploration etc. [2]. Internet technology and small network like WLAN, WPAN, and Mesh gave a doorway for further research to minimize the size and cost of the smart antenna. Now days we have different kind of smart antennas to support even a millimeter wave wireless communication for small network like WPAN or Home network [3].

These advents inefficient hardware ask for a parallel research at logical level implementation. Last ten years have seen many MAC protocols for a network in which all the nodes have been mounted with directional antenna. While my objection is that, the real time scenario will not give us such a unified network in which each node is mounted with directional antenna. For a complete transition of all the devices into a directional antenna supportive device will require one complete wireless generation to pass. We need to develop such a MAC protocol which can satisfy a network needed in the cases of mixture of both types of wireless node (Directional \& Omni). Imagine a real time scenario where you have a wireless local area network composed of different wireless devices. Some of them are new with smart antenna capability and few of them are old. We are sure that the use classic IEEE 802.11 MAC without any modification will cause degradation in the performance. Similarly the use any of the MAC protocol which is devised only for the directional node network it will have a negative impact on the result. And in our work we have shown the negative impact of directional antenna use on different parameter.

This discussion continues in related study, section II. In section III, discussion about how we devised the MAC for directional node, in section IV simulation model and result, section $\mathrm{V}$ concludes the work.

\section{RELATED STUDY}

There have been several works to develop the MAC protocol with directional antenna. We can group directional MAC protocol into two broad categories as i) non-circular DMAC and ii) circular DMAC. Further we can categorize it as follows.
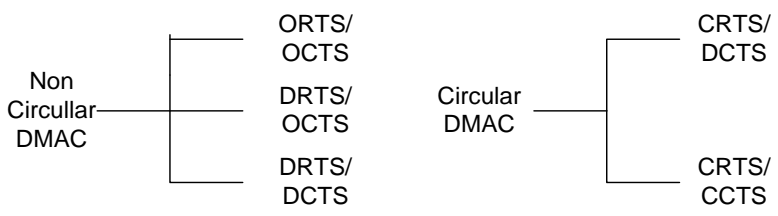

Figure 1.

Category of Directional MAC

First we discuss some of the non circular DMAC. In Nasipuri et [4] al proposed MAC, taken approach is to have multiple non circular directional antenna using which they transmit RTS (ORTS) and CTS (OCTS) in omni mode. Data and ACK is transmitted directionally. Due to Omni mode RTS and CTS, spatial reuse is badly affected. Omni RTS/CTS causes all the over hearer's to stop their transmission.

Ko. YB et al [5] proposed a directional MAC in which location information is determined by the GPS and node share location information with its neighbors. RTS is sent in directional (DRTS) mode because of known location, while CTS are sent in omni mode. Data and ACK is in the pure directional mode. Due to Directional RTS there is slight improvement in spatial 
reuse. Above two protocols suffer a great deal with the deaf node problem.

M Takata et al [6] proposed a directional MAC which they claim for deafness avoidance in the ad hoc network. Hare each device maintains a neighbor table by using the communication history. They introduce a new term wait to send (WTS). This WTS are transmitted after DRTS/DCTS in a circular mode. And hence we can avoid deaf node problems, but cannot enough because WTS is selectively transmitted to a subset of beams. Data and ACK is transmitted in directional mode. Overhead is increased as a tradeoff to throughput. Spatial reuse is increased as compared to above protocols.

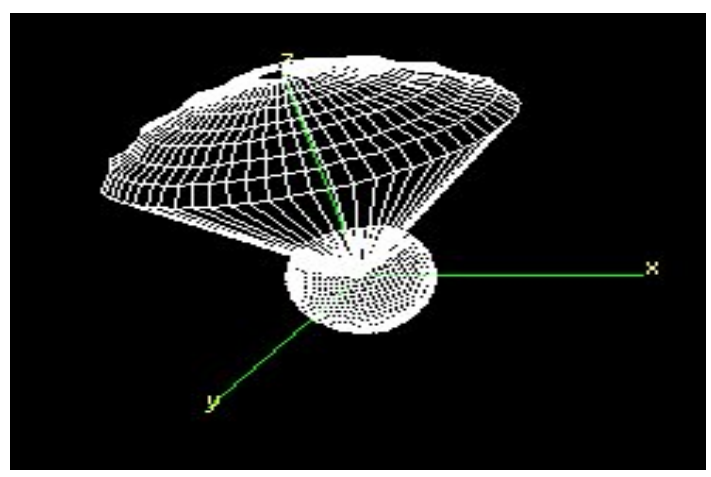

Figure 2.

Directional antenna

Now we discuss few circular directional MAC. T. Korakis et al [7] in their MAC protocol for full exploitation of directional antennas in ad hoc wireless networks they use circular RTS (CRTS) to inform neighbor about ongoing transmission. Each node maintains the location table of neighbors. CTS is sent directionally (DCTS) towards RTS originator. Due to DCTS deafness and hidden node problem increases but due to location table maintenance spatial reuse is good.

G. Jakllari et al [8] present a directional MAC protocol in which they claim to handle asymmetry in gain in directional antenna equipped ad hoc networks. They use circular RTS (CRTS) and circular CTS (CCTS) also but circular CTS are partially transmitted to a non overlapping region of CRTS to reduce overhead. Location table is also maintained on the base of source beam, destination beam and node ID. CRTS and CCTS reduces deafness and hidden node problems.

None of the above protocol cares about a situation where we can have a mixture of omni and directional antenna patterned wireless node. Although it is quite an important issue because the wireless market is not going to switch in a night to a complete directional antenna facilitated devices. In this work we have not proposed any solution for this sort of scenario where we can manage a mixture of omni and directional wireless devices. We did a performance evaluation of a mix scenario under IEEE 802.11 standard parameters, and found that if we do not have any proper MAC to solve this issue we will get degraded output in spite of upgrading it.

\section{SIMULATION ENVIRONMENT}

When The simulation is done using Optimized Network Engineering Tools (OPNET) to characterize the network performance. Pipeline stages have been used to model the communication channel, which includes antenna gains, signal to noise ratio, propagation delay, transmission delay, different kind of noise etc. this tool enables designers to create an almost realistic scenario.

This simulation tool provides an antenna pattern editor using which we can devise the directionality and the gain. In our scenario nodes are of mixed nature, few of them using directional antennas and the remaining nodes uses an isotropic antenna. Figure 2 shows the designed antenna having conical shape and bandwidth of $2 \pi / 4$ radian. It has $10 \mathrm{dBi}$ gain for main beam and $-20 \mathrm{dBi}$ for the side lobes. Nodes use omni directional mode while receiving and for transmission it uses directional mode. The antenna is adaptive in nature; it means it can rotate itself to transmit data to nodes in any direction. Nodes are capable of interchange the mode with negligible latency. When antenna is in omni mode its gain is $\mathrm{G} 0$, and in directional mode it is Gd, where Gd > G0.Due to focus of antenna in one direction coverage range is increased.

In OPNET we modified the node model and process model of IEEE 802.11 to support the directionality of the nodes. For omni directional nodes the inbuilt MAC process model is used intact. In the node model of directional node, one antenna pattern is added to the node model and MAC process model is modified as per requirement. Their working is implemented in the function block of the MAC process model. For getting the location of the destination node a global array is initialized. MAC id of the node is used as index of the array and location is stored on each index. We can see the little modified as well as non modified node model in Figure 3.

These node models are associated with the nodes as per our scenario demands. We arranged the 16 nodes as figure 4 and designed two scenario. In first scenario all nodes are in omni directional whereas, in second scenario eight nodes are in omni directional and eight are directional nodes. The area of scenario is $500 \mathrm{~m} \times 500 \mathrm{~m}$. Each simulation run for a period of 10 simulation minutes and it is averaged over 128 different seeds. While receiving, directional node uses omni mode and for transmission they take advantage of the directionality. There is no specific protocol associated with their working. In Table 1 simulation parameters are specified for both the scenarios. 


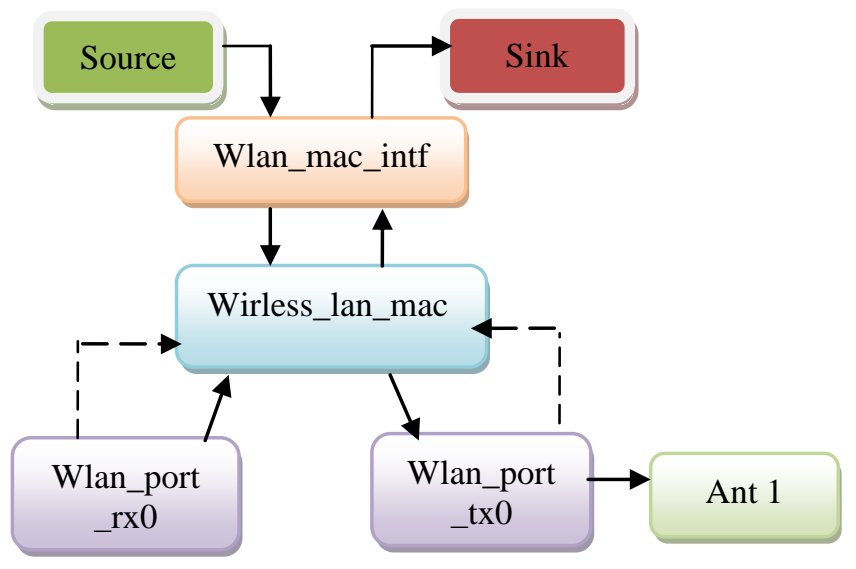

Node Model for Direction Nodes

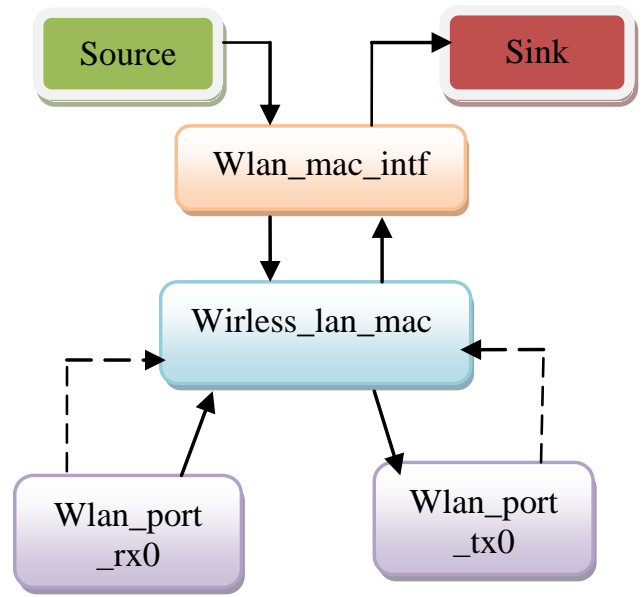

Node Model for Omni Directional Nodes

Figure 3. Node Model

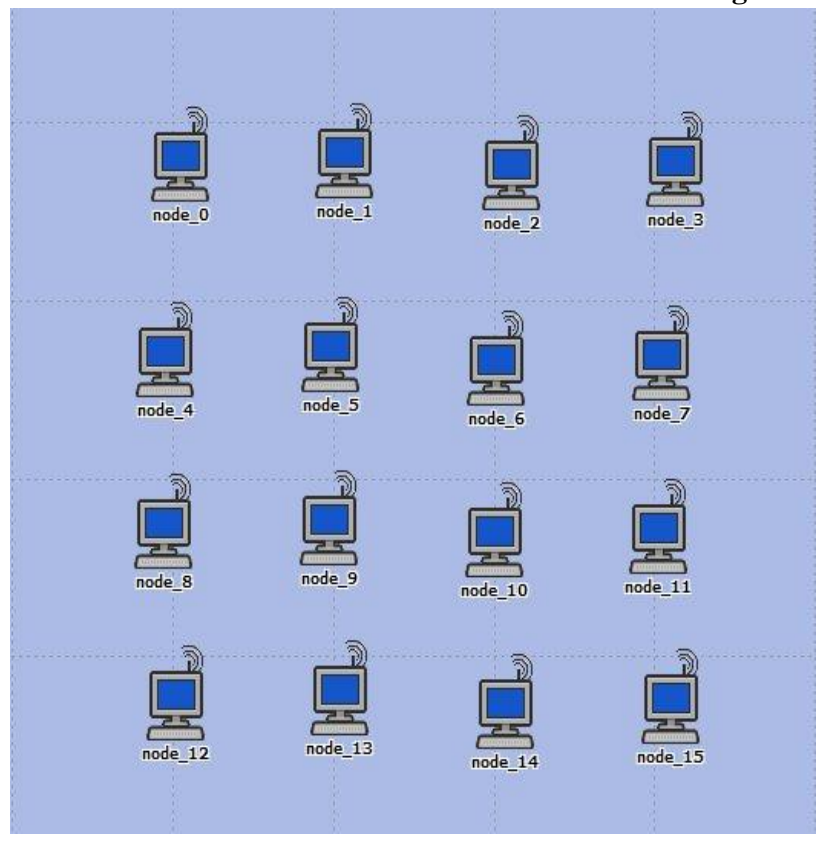

Figure 4. Simulation Scenario

TABLE 1. SIMULATION PARAMETER

\begin{tabular}{|l|l|}
\hline \multicolumn{1}{|c|}{ Parameters } & \multicolumn{1}{c|}{ Values } \\
\hline Network Scale & Campus \\
\hline Area & 500 X 500 meters \\
\hline Number of Node & 16 \\
\hline Directional Node & 8 \\
\hline Omni Node & 8 \\
\hline Directional gain & $10 \mathrm{dBi}$ \\
\hline Packet Interval (Sec) & Exponential (1.0) \\
\hline Packet Size (byte) & 1024 \\
\hline Simulation Time & $10 \mathrm{~min}$ \\
\hline Node Type & Fixed \\
\hline $\begin{array}{l}\text { Reception power } \\
\text { Threshold }\end{array}$ & $-95 \mathrm{dBm}$ \\
\hline Transmission power & $0.005 \mathrm{~W}$ \\
\hline
\end{tabular}

\section{PERFORMANCE EVALUATION}

In this section we evaluate the simulation results in terms of aggregate throughput and end to end delay (latency). Throughput is defined as the average rate of successful message delivery over a communication channel. The graph of end to end delay and the throughput comparison depicted in Figure 5 and Figure 6 respectively. we can see in the figures, that mixed node (Direct-Omni) scenarios' throughput is lower than the omni mode. The average delay in the both scenarios is the reverse of the throughput because delay is inversely proportional to the throughput. Delay in the case of direct Omni mode is larger than the pure omni mode. As we discussed previously that new MAC has been proposed for the pure directional node but not for a network where we can find a group of mixture of the node (Directional and Omni directional) mode.

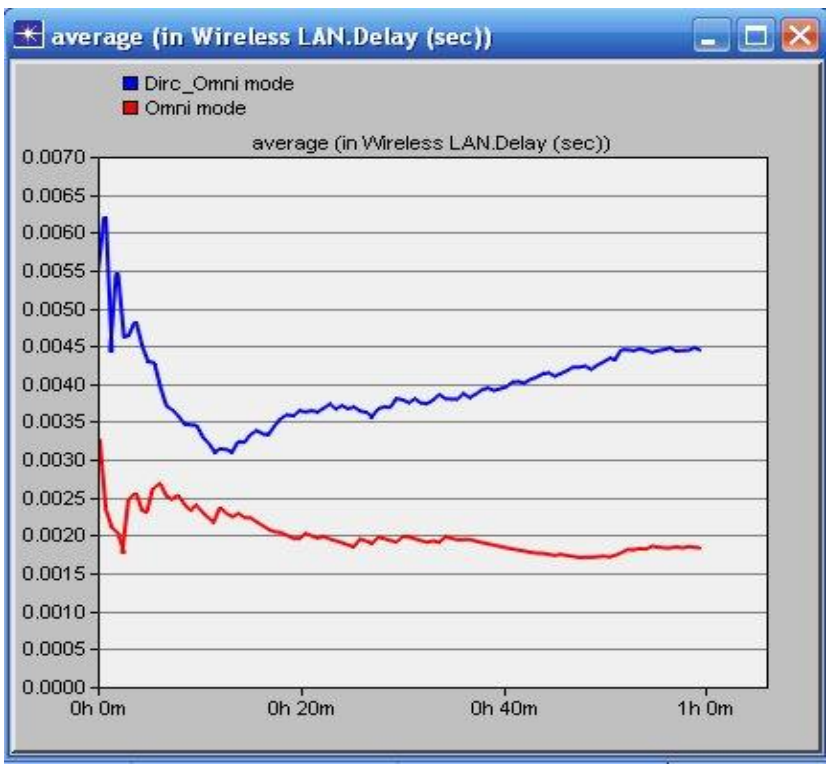

Figure 5. End to End Delay 


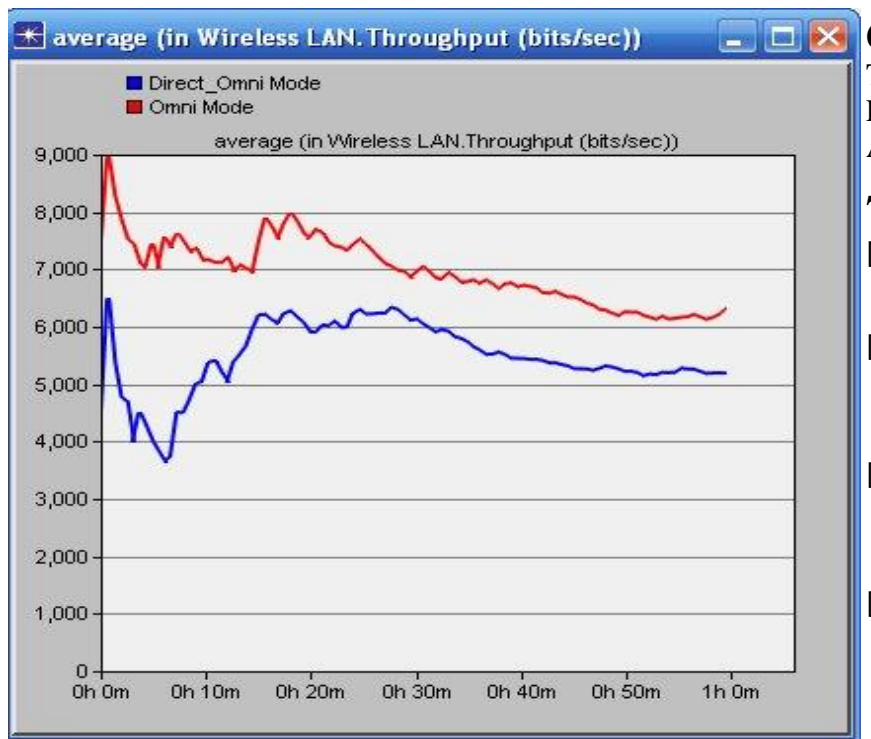

Figure 6. Average Throughput

It is quite possible that an ad hoc network may allow a directional antenna oriented device unknowingly. Although the network will work but it will suffer a great deal of performance which we can see here. Both the performances are degrading in the case of a mixture of both kinds of devices.

\section{CONCLUSIONS}

This work aims to find a drawback in our approach taken to use a directional antenna to improve performance of the ad hoc network. Getting all the nodes of an ad hoc network in a directional antenna mode is difficult. In a real time scenario we are bound to face a network with a mixture of directional and Omni node, where we will need an efficient protocol so that we can enhance the overall capability of the network. Otherwise use of directionality in a node is not going to boost overall performance. Our simulation result shows the comparison of both the scenario which pushes us to devise fresh protocols for mixed scenario.

\section{ACKNOWLEDGMENT}

This work was supported by college of e-Learning \& Distance Learning Deanship, King Saud University, Riyadh, Saudi Arabia.

\section{REFERENCES}

[1] Radio/Wireless Models, OPNET, http:/www.opnet.com/, 2010.

[2] B.D. Van Veen, K.M. Buckley, "Beamforming: A Versatile Approach to Spatial Filtering," IEEE ASSP Magazine, vol. 5, issue. 2, pp 4-24, April 1988.

[3] K.C. Huang, D.J. Edwards , "Millimeter wave antennas for gigabit wireless communications," Willey,c2008, Hoboken, NJ, USA.

[4] A.Nasipuri, S.Ye,J.You, R.E.Hiromoto, "A MAC protocol for mobile ad-hoc networks using directional antennas", In Proceedings of IEEE Wireless Communications and Networking Conference(WCNC), Chicago, I L, 2000.

[5] YB Ko, V Shankarkumar, and NH Vaidya, "Medium access control protocols using directional antennas in ad hoc networks," INFOCOM 2000, Tel Aviv, Israel, 2000, pp. $13-21$.

[6] M. Takata, M. Bandai and T. Watanabe, "MAC Protocol with Directional Antennas for Deafness Avoidance in Ad Hoc Networks," Proc. IEEE GLOBECOM, pp.620-625, 2007.

[7] T. Korakis, G. Jakllari, and L. Tassiulas, "A A MAC protocol for full exploitation of Directional Antennas in Ad-hoc Wireless Networks", Proc. ACM MobiHoc 2003, 2003.

[8] G. Jakllari, J. Broustis, T. Korakis,S V. Krishnamurthy, and L. Tassiulas, "Handling Asymmetry in Gain in Directional Antenna Equipped Ad Hoc Networks," Proc. IEEE 16th International Symposium on PIMRC, pp.12841288. 